\section{Stacked Patch Antenna with Dual-Polarization and Low Mutual Coupling for Massive MIMO}

\author{
Yue Gao, Runbo Ma, Qianyun Zhang and Clive Parini
}

\begin{abstract}
Massive MIMO has attracted significant interests in both academia and industry. It has been considered as one of most promising technologies for 5G wireless systems. The largescale antenna array for base stations naturally becomes the key to deploy the Massive MIMO technologies. In this paper, we present a dual-polarized antenna array with 144 ports for Massive MIMO operating at $3.7 \mathrm{GHz}$. The proposed array consists of 18 low profile sub-arrays. Each sub-array consists of 4 single units. Each single antenna unit consists of one vertically polarized port and one horizontally polarized port connected to power splitters, which serve as a feeding network. A stacked patch design is used to construct the single unit with the feeding network, which gives higher gain and lower mutual coupling within the size of a conversional dual-port patch antenna. Simulation results of the proposed single antenna unit, sub-array and Massive MIMO array are verified by measurement.
\end{abstract}

Index Terms-MIMO, antenna array, sub-array, Massive MIMO, mutual coupling, dual-polarization, feeding network.

\section{INTRODUCTION}

Multiple-Input and Multiple-Output (MIMO) has been a very important technique for wireless communications, such as $3 \mathrm{G}, 4 \mathrm{G}$, WiFi, etc. Enormous research has been carried out for MIMO terminal antennas addressing challenges of limited space in handheld devices [1]-[4]. Driven by the development of 5G wireless systems, Massive MIMO recently has attracted significant interests in both academia and industry as it has shown over 10 times the spectral efficiency increase than a conventional MIMO under realistic propagation environment conditions and simpler signal processing algorithms [5] [6].

With over 100 antenna ports to be implemented at the base station, Massive MIMO has brought significant challenges. An active multibeam antenna system for 5G Massive MIMO wireless system was designed and implemented in [7], which contained $64 \mathrm{RF}$ Channels and 64 elements array being divided between 8 PCBs. The dimension of each individual PCB was $320 \mathrm{~mm} \times 215 \mathrm{~mm}(6 \lambda \times 4 \lambda$, where $\lambda$ is the wavelength in free space). The antenna array resonated at $5.8 \mathrm{GHz}$ and the impedance bandwidth was $200 \mathrm{MHz}$. The simulated and measured gain of the antenna array reached $13 \mathrm{dBi}$ and $11 \mathrm{dBi}$, respectively. A system design by integrating an EM lens with the large antenna array was proposed in [8], which had the capability of focusing the power of any incident plane wave passing through the EM lens to a small focal area of the antenna array, depending on the angle of arrival of the wave. In [9], a practical 2D active patch antenna array configuration for Full Dimension MIMO systems was proposed. A patch antenna sub-array with $1 \times 4$ elements had a gain of $11.7 \mathrm{dBi}$.

Yue Gao, Qianyun Zhang and Clive Parini are with Queen Mary University of London, London, United Kingdom (e-mail: \{yue.gao, qianyun.zhang, c.g.parini\}@qmul.ac.uk.)

Runbo Ma is with Macao Polytechnic Institute - QMUL Information Systems Research Centre (ISRC), Rua de Gomes, SAR Macao (e-mail: r.ma@qmul.ac.uk).
The MIMO array comprised of $8 \times 4$ such sub-arrays occupying an area of $4 \lambda \times 8 \lambda$, which provided an array gain of $15 \mathrm{dBi}$ in simulation.

Stacked patch antennas in [10]-[14] showed a low-profile antenna structure. The patch fed by orthogonal slots in [11][13] demonstrated a dual-polarization with high isolation. The capacitive coupling feeding was further explored in [14]. However, those antenna structure must be equipped with two exciting structures for each patch in order to achieve the dualpolarization. In this paper, a low profile stacked patch antenna unit is designed with four apertures through four coupling strips to excite four patches with the dual-polarization. Each aperture transfers the input power to its crossed coupling strip without any via, and then each coupling strip excites two patches. The proposed antenna unit is designed as a symmetric layout in layers except the feeding network at the bottom layer, resulting in advantages such as simple structure and low mutual coupling between the two orthogonal polarization modes. A sub-array consisting of 4 single antenna units is also proposed to achieve higher gain. The measured mutual coupling and radiation patterns of the sub-array at $3.7 \mathrm{GHz}$ are compared with simulated ones. Furthermore, 18 sub-arrays are fabricated to construct a Massive MIMO array with 144 ports and 288 patches. The array configuration, which takes the form of a Turning Torso of three stacked orthohexagonal rings within a volume of $648 \mathrm{~mm} \times 648 \mathrm{~mm} \times 258 \mathrm{~mm}(8 \lambda \times 8 \lambda \times 3 \lambda)$ is presented. Measurement shows that the mutual coupling between any two ports in the array is lower than $-35 \mathrm{~dB}$.

\section{Proposed Single Antenna Unit}

\section{A. Structure and operating principle}

The proposed single antenna unit utilizes a planar structure with 7-stacked layers, as shown in Fig. 1. Layer 1, 3, 5 and 7 are metal layers and Layer 2, 4 and 6 are dielectric substrate layers. With Rogers RT5880 as an example, the dielectric constant is $\epsilon_{r}=2.2$ and loss tangent is $\delta=0.0009$. The single antenna unit operating at $3.7 \mathrm{GHz}$ with height of $h_{1}=(1 / 2) h_{2}=h_{3}=0.762 \mathrm{~mm}$ is optimized in CST Microwave Studio. On Layer 1, four square radiating patches with a side length of $l_{1}=23.7 \mathrm{~mm}$ are arranged in a $2 \times 2$ matrix form. The space between the edges of adjacent patches is $s_{e}=13.7 \mathrm{~mm}$ (about $0.17 \lambda$ ). On Layer 3, four metallic coupling strips with length of $l_{a}=16.6 \mathrm{~mm}$ are placed under the middle of the space between adjacent patches, and the two open ends of each strip are overlapped by the two adjacent patches. On Layer 7, a feeding network comprised of two Tjunction power splitters transfers power from Port 1 and 2 to the four coupling strips via four bow-tie apertures on Layer 5 . The structure layouts on Layer 1, 3 and 5 are symmetric with respect to the $\mathrm{x}$-axis and $\mathrm{y}$-axis. The size of the antenna unit is $81 \mathrm{~mm} \times 86 \mathrm{~mm}$ (about $\lambda \times \lambda$ ).

The power coming from one of the two port is split into two equal ones which are guided to the two bow-tie apertures on the ground, and then coupled to the metallic coupling strips above the apertures. With the symmetric structure, the maximum magnitude of the coupled current and zero magnitude of E-field occur at the middle of each coupling strip, 


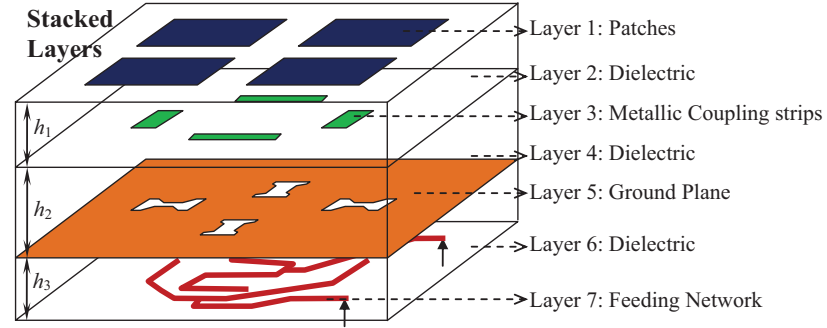

(a)

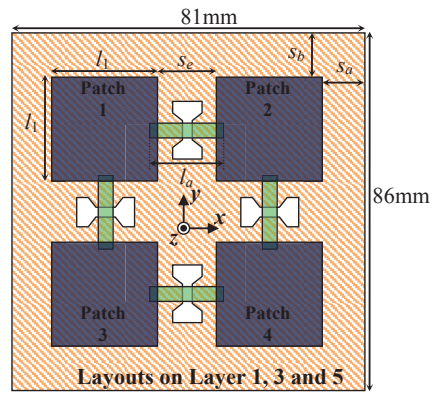

(b)

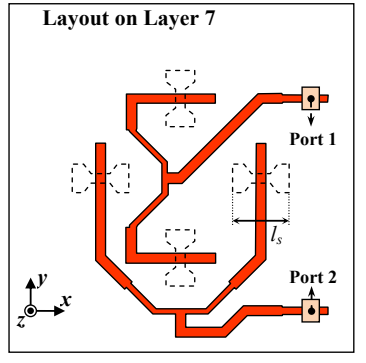

(c)
Fig. 1. Structure of the proposed antenna unit: (a) prospective view, (b) layouts on Layer 1, 3 and 5, and (c) layout on Layer 7. (With $h_{1}=\frac{1}{2} h_{2}=$ $h 3=0.762, l_{1}=w_{1}=23.7, l_{a}=16.6, s_{e}=13.7, s_{a}=10.0$ and $s_{b}=12.5$, all dimensions are in $\mathrm{mm}$ ).

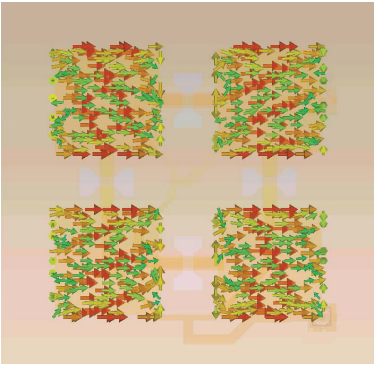

(a)

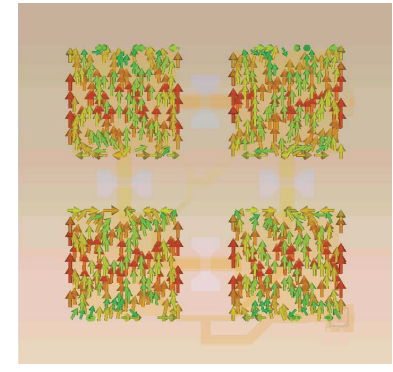

(b)
Fig. 2. Surface currents on the four patches: (a) horizontal linear polarization excited by Port 1, and (b) vertical linear polarization excited by Port 2 .

while E-fields around both open ends of each coupling strip have identical amplitudes but opposite phases. Each set of the splitter end, aperture and coupling strip acts as a transformer balun that drives the two radiating patches by capacitive coupling at the two ends of coupling strip overlapped by the patches. Therefore, Port 1 excites stable and in-phase currents along the $\mathrm{x}$-axis on all four patches, and Port 2 excites stable and in-phase currents along the y-axis, as shown in Fig. 2. The horizontal or vertical polarization $T M_{01}$ modes of four patches are created independently by Port 1 or 2 .

The symmetric layouts on Layer 1, 3 and 5 are beneficial to increase the linearity of each polarization. This is because when one polarization mode is excited, the amplitude of Efield at the mid-point of the non-radiating edge of each patch, just above the coupling strip end for the other polarization mode, is almost zero due to the symmetric layouts. Furthermore, the patches and feeding network are separated by the ground plane. The last segments of the horizontal and vertical

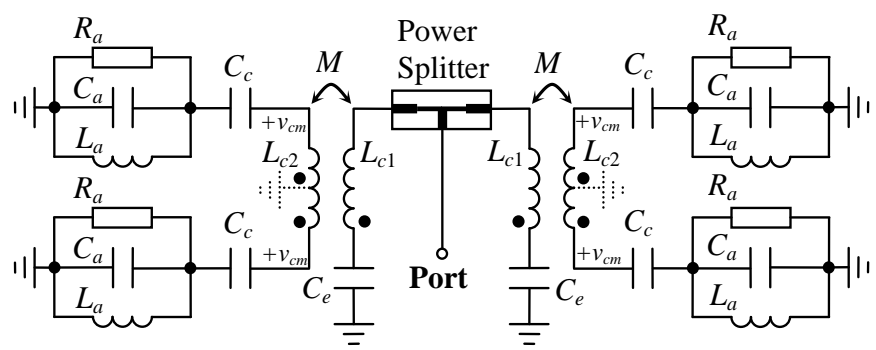

Fig. 3. Equivalent circuit of the antenna unit for any port.

power splitters are orthogonal which leads to he low mutual coupling level between Port 1 and 2. Each polarization mode of the proposed antenna unit are operating independently, and therefore it can be expressed by the same equivalent circuit given in Fig. 3. The four same $L_{a} C_{a} R_{a}$ shunt circuits represent the four same radiating patches operating around the resonant frequency. Components $L_{c 1}, L_{c 2}, \mathrm{M}$ (magnetic coupling between $L_{c 1}$ and $L_{c 2}$ ), $C_{e}$ and $C_{c}$ stand for the transformer balun and the capacitive coupling between coupling strip and patches.

$L_{c 2}$ in the equivalent circuit represents the corresponding coupling strip, and the middle of $L_{c 2}$ is a virtual ground. Hence, the $L_{a} C_{a} R_{a}$ shunt circuit is loaded with the series circuit comprised of $C_{c}$ and half of $L_{c 2}$. Because the series resonant frequency of $L_{c 2} C_{c} / 2$ is far higher than the shunt resonant frequency of $L_{a} C_{a}$, the $L_{c 2} C_{c} / 2$ series circuit presents capacitive character around the shunt resonant frequency of $L_{a} C_{a}$. As a result, resonant frequency of the antenna unit can be slightly decreased and controlled by adjusting $C_{c}$ and $L_{c 2}$, which is corresponding to controlling the length of the coupling strip, $l_{a}$.

The other advantage of the symmetric layout of the proposed antenna unit is the ability to eliminate the mutual coupling between the horizontal and vertical polarization modes. As shown in Fig. 2, even if there are mutual coupling signals on the two ends of each coupling strip exciting the vertical polarization mode when the horizontal polarization mode is being excited, the signals are same amplitude and in-phase because of the symetric layout, thus each of the vertical coupling strip is excited by the horizontal mutual coupling signals in common mode. It can be seen in Fig. 3 that the common mode mutual coupling signals, labeling as $+v$, cannot generate current in $L_{c 2}$, so that the mutual coupling of the horizontal mode is eliminated in the vertical coupling strip. In the same way, when the vertical polarization mode is being excited, each of the horizontal coupling strips is excited on its both ends by the vertical mutual coupling signals in common mode, and the mutual coupling of the vertical mode is eliminated in the horizontal coupling strip. This is a positive feature to enhance the isolation for the dual-polarization.

\section{B. Simulated and measured results}

Without any via inside the stacked structure, the proposed antenna unit is easy to be fabricated by using multilayer planar structures. A prototype of the proposed single antenna unit is fabricated in a multi-layer stacked patch as shown in Fig. 4. 


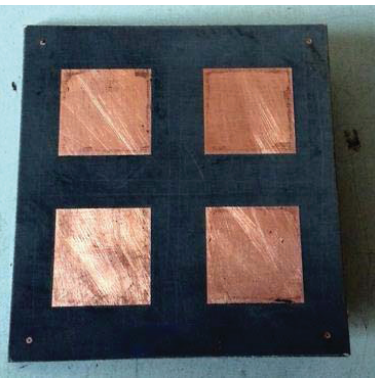

(a)

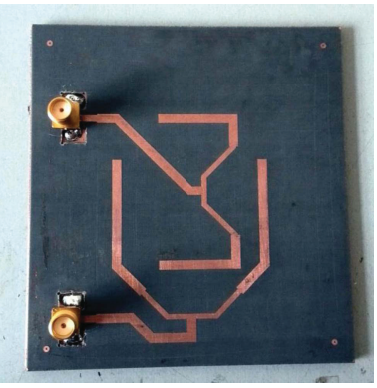

(b)
Fig. 4. The prototype of the single antenna unit: (a) front view and (b) back view.

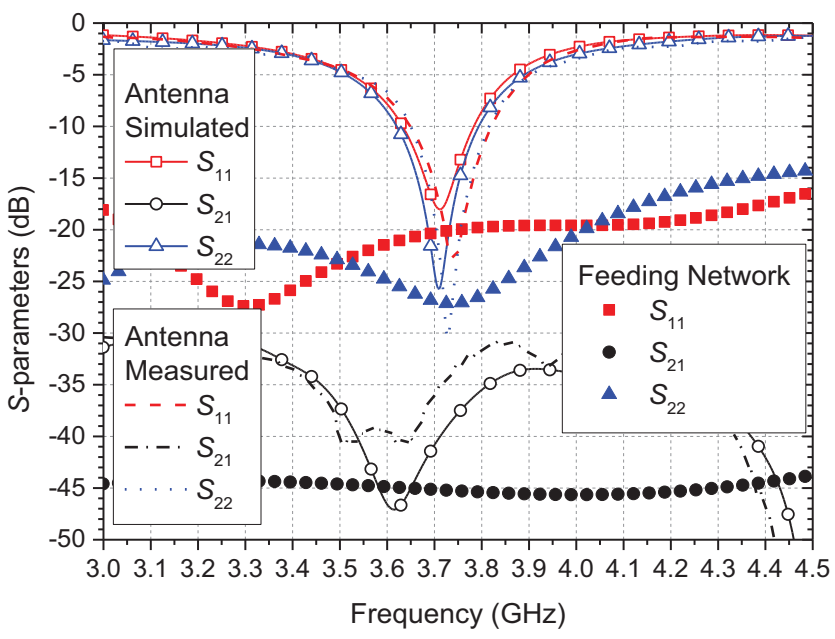

Fig. 5. The measured and simulated S-parameters of the antenna unit and its feeding network with matching terminators.

The simulated S-parameters are compared with measured ones in Fig. 5. The measured -10dB return loss of the antenna unit is from $3.65 \mathrm{GHz}$ to $3.81 \mathrm{GHz}$ with impedance bandwidths of $160 \mathrm{MHz}$, which matches well with the simulations. It can be seen that the simulated and measured $S_{21}$ between two exciting ports are less than $-33 \mathrm{~dB}$ and $-31 \mathrm{~dB}$ respectively, which verifies the mutual coupling level between the two polarization exciting ports is very low. This low mutual coupling benefits from the symmetric and multilayer structure of the antenna unit, where the two excited linear polarization modes are orthogonal. Additionally, the mutual coupling between the two power splitters with matching terminators is also given in Fig. 5, and is less than $-45 \mathrm{~dB}$, which supports the low mutual coupling between the two ports.

Fig. 6 gives the measured and simulated radiation patterns at 3.7GHz. The radiation patterns of horizontal and vertical linear polarization are very similar. For the horizontal linear polarization, the measured half power beam widths are about $52^{\circ}$ and $55^{\circ}$ in E-plane and H-plane respectively. For the vertical linear polarization, the measured ones are about $54^{\circ}$ and $53^{\circ}$ in E-plane and H-plane respectively. For both the polarization, the front-back ratios are greater than $15 \mathrm{~dB}$, and the gain of cross-polarization are $25 \mathrm{~dB}$ lower than the co-polarization at the boresight. The realized gains at boresight with respect to frequency in the co-polarized and cross-polarized planes are

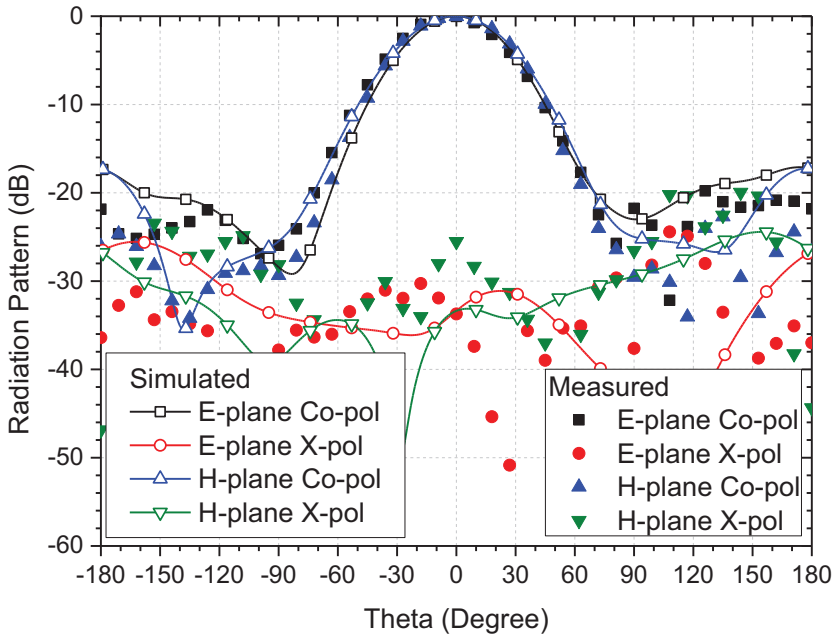

(a)

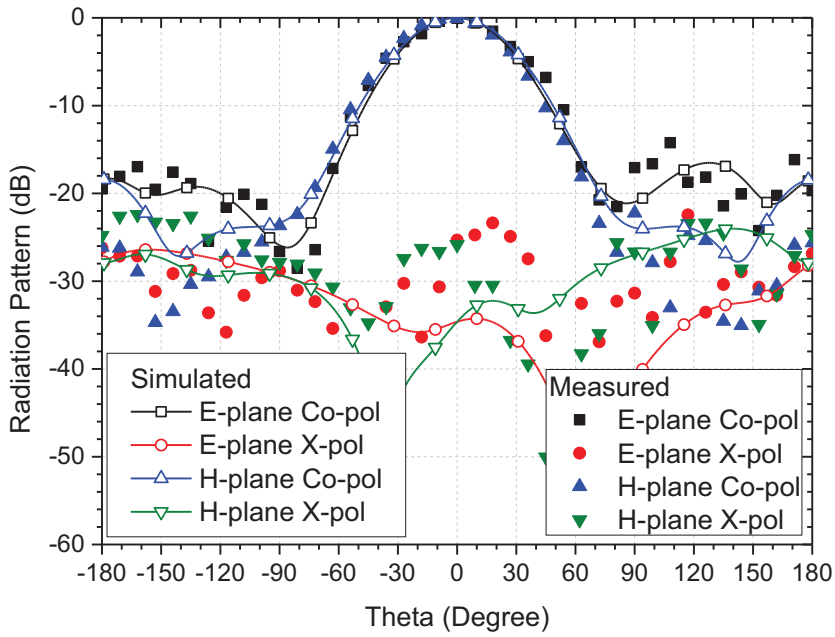

(b)

Fig. 6. The measured and simulated radiation patterns at $3.7 \mathrm{GHz}$, (a) horizontal linear polarization, (b) vertical linear polarization.

given in Fig. 7. The measured gains of both linear polarization are about $10.5 \mathrm{dBi}$ at $3.7 \mathrm{GHz}$. From $3.65 \mathrm{GHz}$ to $3.84 \mathrm{GHz}$, copolarized gains are higher than $10 \mathrm{dBi}$, and are $23 \mathrm{~dB}$ higher than cross-polarized ones. It verifies that the antenna unit has good linearity in both polarization.

\section{Proposed Sub-array and Massive Mimo ANTENNA ARRAY}

\section{A. Sub-array with $1 \times 4$ antenna units}

In order to flexibly build a large-scale array for the Massive MIMO, a sub-array with $1 \times 4$ antenna units is designed by arranging the proposed antenna units along $\mathrm{x}$-axis directly, as shown in Fig. 8, occupying a size of $324 \mathrm{~mm} \times 86 \mathrm{~mm}(4 \lambda \times \lambda)$. There are 4 ports in the sub-array for each linear polarization mode. The space between two adjacent patches is only $2 s_{a}=$ $20 \mathrm{~mm}$ (e.g. $0.25 \lambda$ ).

The simulated and measured S-parameters of a sub-array are shown in Fig. 9. Compared with the simulated return loss in Fig. 5, it is found that the center frequencies of the subarray are slightly lower than those of the single antenna unit, 


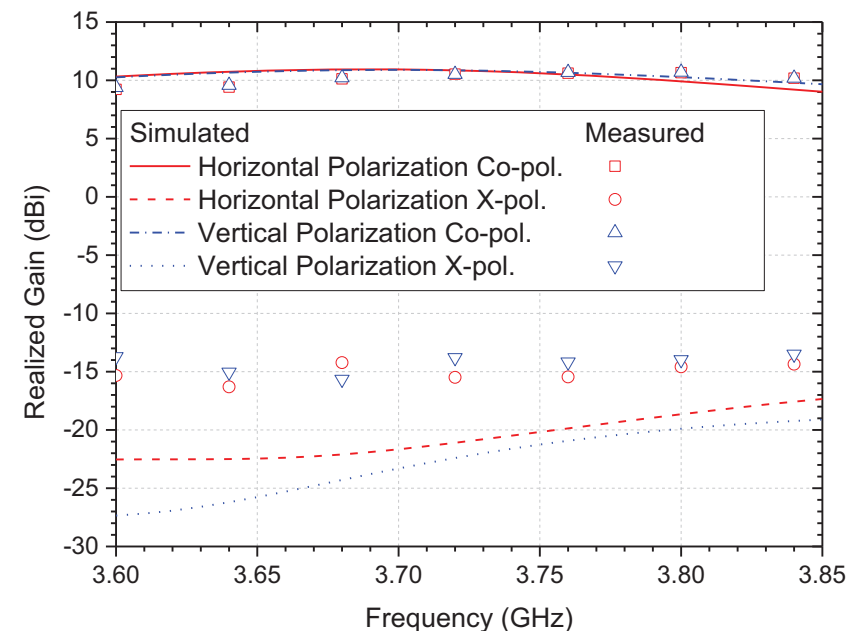

Fig. 7. The measured and simulated realized gains with respect to frequency at boresight.

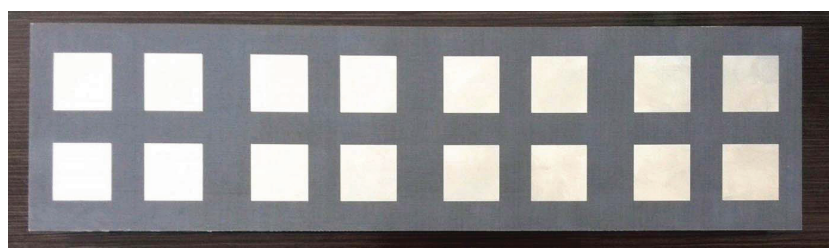

(a)

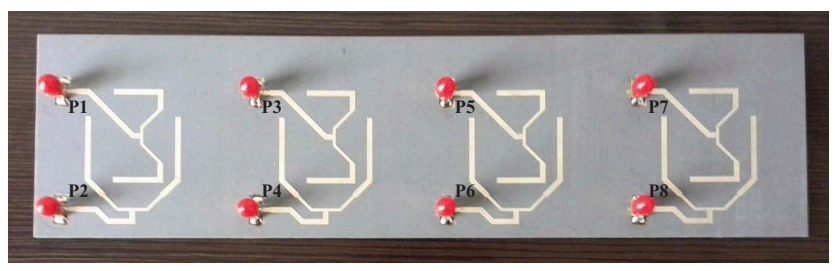

(b)

Fig. 8. The sub-array with four antenna units: (a) front view of the fabricated sub-array and (b) back view of the fabricated sub-array.

because the equivalent area of substrate for each unit in the sub-array becomes slightly larger. As shown in Fig. 9 (a), the $-10 \mathrm{~dB}$ bandwidth of $160 \mathrm{MHz}$ is achieved for all ports, which is from $3.65 \mathrm{GHz}$ to $3.81 \mathrm{GHz}$. The representative measured mutual coupling levels between ports are given in Fig. 9 (b). It can be seen that the maximum mutual coupling levels occur between any adjacent ports and are less than $-32 \mathrm{~dB}$ within the bandwidth. The mutual coupling levels between non-adjacent ports are less than $-40 \mathrm{~dB}$.

As shown in Fig. 10, the sub-array features a directional radiation pattern with maximum realized gain of $16.7 \mathrm{dBi}$ along the normal of the sub-array face when the four ports for the same linear polarization are excited in-phase. For horizontal linear polarization, the zx-plane is the E-plane and yz-plane is the H-plane, while for vertical linear polarization, yz-plane is the E-plane and zx-plane is the H-plane. Because the antenna units are arrange along $\mathrm{x}$-axis, the half power beam widths for both polarization modes on the zx-plane are about $13^{\circ}$, much narrower than the single antenna unit. While the half power beam widths of both polarization modes on yz-plane are about

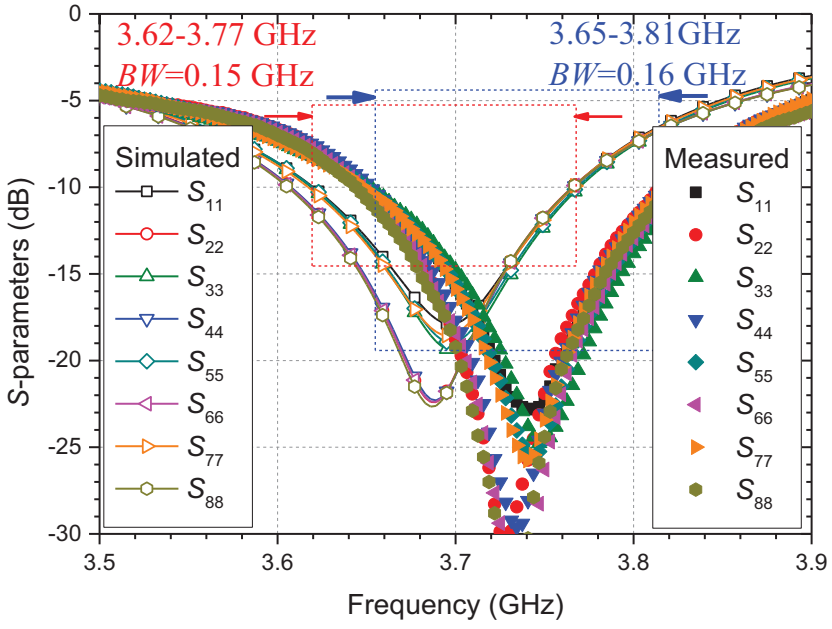

(a)

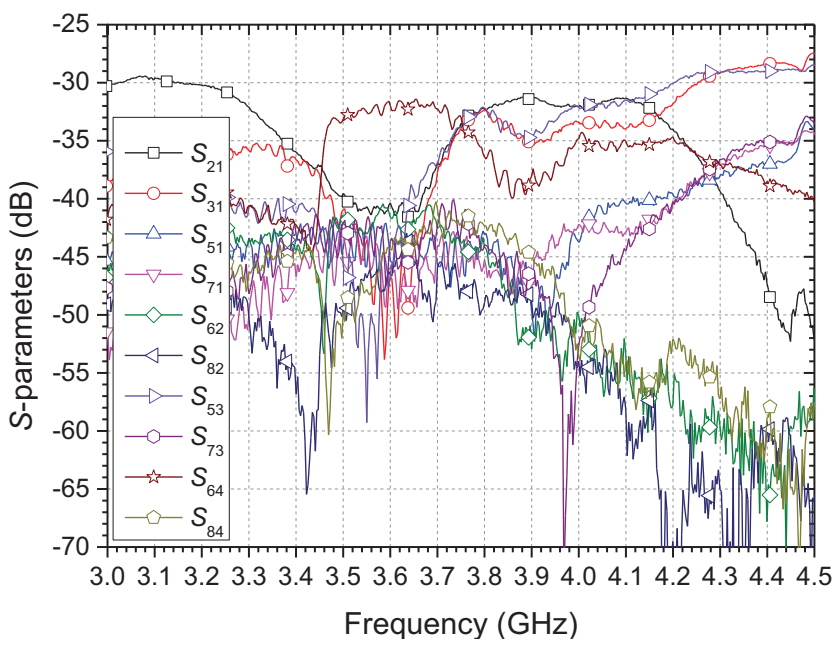

(b)

Fig. 9. The S-parameters of the sub-array: (a) return loss and (b) mutual coupling.

$53^{\circ}$, which is same as the single antenna unit.

\section{B. Mutual Coupling Analysis}

The sub-arrays can be arranged flexibly in different manners to build a larger array, for example in matrix to build a planar array. In order to efficiently analyze the mutual coupling between different antenna units, a simple planar array with $2 \times 2$ units are modeled and studied, as shown in Fig. 11 (a). The planar array with ports labeled from P1 to P8 is simulated with two kinds of boundary conditions, e.g. open boundary and periodic boundary. The mutual coupling levels obtained in the two conditions can be treated as the approximated ones between adjacent antenna units arranged along the edge and in the inner area of a large array.

The mutual coupling levels of this planar array are given in Fig. 11 (b). Almost every mutual coupling level under periodic boundary conditions is higher than its corresponding one under open boundary conditions. It is reasonable to assume these two curves as the upper and lower bounds for the real mutual coupling level between the same ports. 


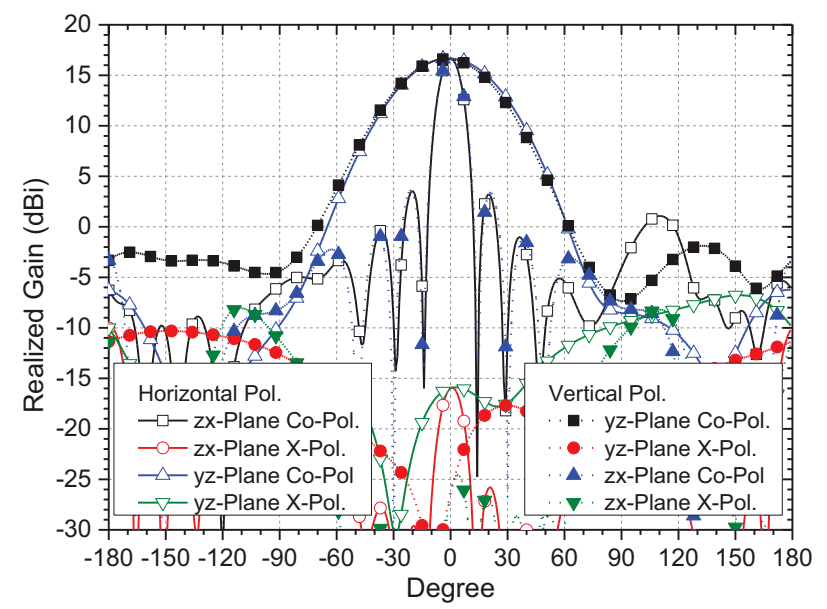

Fig. 10. The radiation patterns of the sub-array excited with synchronous signals in all 4 ports for horizontal polarization or vertical polarization.

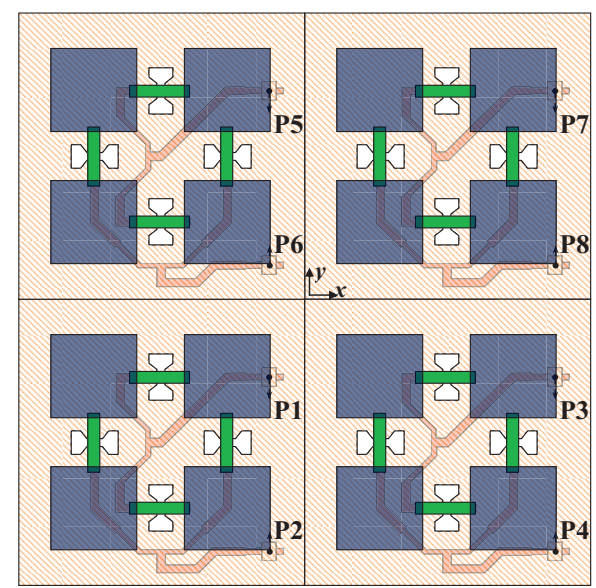

(a)

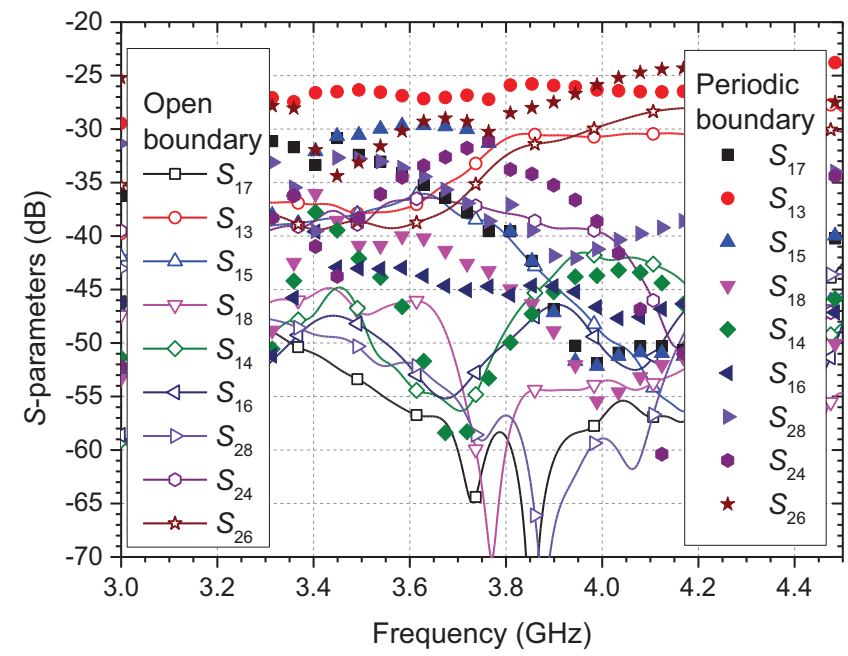

(b)

Fig. 11. (a) $2 \times 2$ units for mutual coupling study and (b) the mutual coupling levels of the array with $2 \times 2$ units under different boundary conditions.

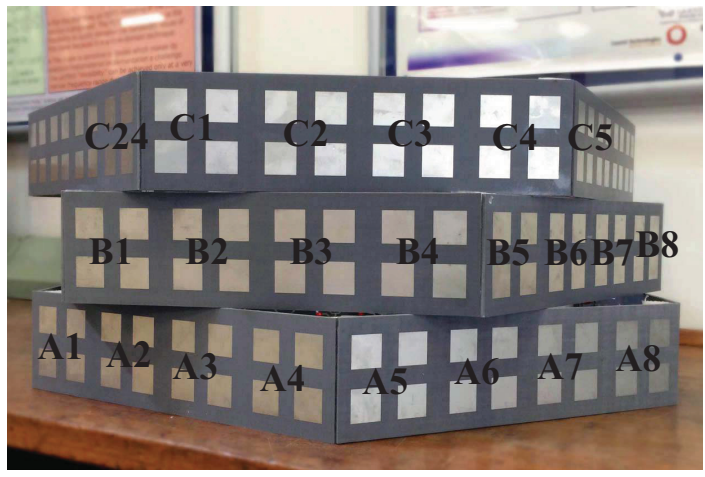

(a)

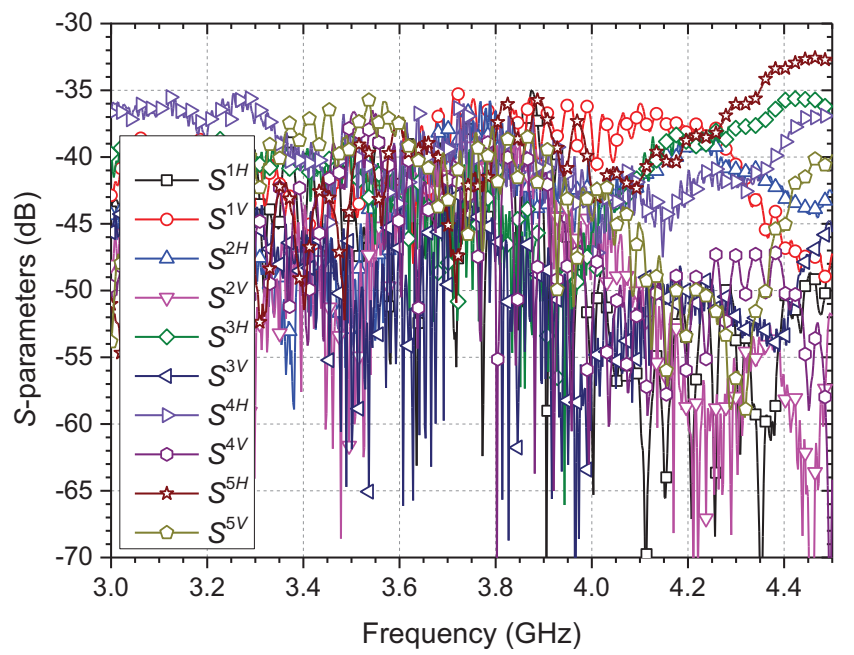

(b)

Fig. 12. (a) The configuration of three level Turning Torso antenna array and (b) mutual coupling levels.

Based on the exciting polarization and antenna unit arrangement, the mutual coupling between different ports is categorized into 4 classes:

1) Class-A mutual coupling - the highest two levels are $S_{13}$ and $S_{26}$. As shown in Fig. 11 (a), the highest mutual coupling occurs between the ports exciting the same polarization in the two adjacent antenna units along the excited polarization direction. The mutual coupling level is ranging from $-35 \mathrm{~dB}$ to $-25 \mathrm{~dB}$.

2) Class-B mutual coupling - the second high mutual coupling levels include $S_{15}$ and $S_{24}$ and occurs between the ports exciting the same polarization in the two adjacent antenna arranged perpendicularly to the excited polarization direction, ranging from $-40 \mathrm{~dB}$ to $-30 \mathrm{~dB}$.

3) Class-C mutual coupling - this kind of mutual coupling level occurs between the ports exciting the same polarization and belonging to the antenna units arranged diagonally, including $S_{17}$ and $S_{28}$, ranging from -50dB to $-35 \mathrm{~dB}$.

4) Class-D mutual coupling - the lowest mutual coupling levels include $S_{14}, S_{16}$ and $S_{18}$ and occur between the ports exciting different polarization and belonging to different antenna units. The mutual coupling level is always less than $-40 \mathrm{~dB}$. 


\section{Proposed Massive MIMO antenna array}

For the Massive MIMO BS system, an array configuration providing 18 independent beams is fabricated, as shown in Fig. 12 (a). The array configuration is based on the Turning Torso building architecture, consisting of three stacked stages of orthohexagonal wall with a progressive twisting angle of $20^{\circ}$ between adjacent stages. Each stage is composed of six sub-arrays. Therefore, 18 sub-arrays are distributed around the whole circumference, the radius of which is about $4 \lambda$. The configuration reduces the radial size of the array by increasing the longitudinal vertical size of the stack. The whole array has a volume of $648 \mathrm{~mm} \times 648 \mathrm{~mm} \times 258 \mathrm{~mm}$ (about $8 \lambda \times 8 \lambda \times 3 \lambda$ ), which consists of 288 patches and 144 ports.

The three stacked stages are labeled A, B and C, in which there are totally 24 antenna units labeled with the sequential number from 1 to 24 in order to facilitate the following mutual coupling analysis. Extensive measurement is carried out to study the mutual coupling between antenna units belonging to the different sub-arrays. For mutual coupling between antenna units at different sub-arrays, it is found that the relatively high mutual coupling levels occur between the adjacent antenna units, which can be divided into five types of unit pairs. The vertically adjacent pairs of unit B1 and C24, B2 and C1, B3 and $\mathrm{C} 2, \mathrm{~B} 4$ and $\mathrm{C} 3$ and horizontally adjacent pair of unit $\mathrm{C} 1$ and $\mathrm{C} 24$ can stand for the five types. The measured mutual coupling of the five types is given in Fig. 12 (b) as $S^{n H(\text { orV })}$, in which the superscript $n$ sequentially indicates the 5 types of unit pairs, and $\mathrm{H}$ (or V) indicates the mutual coupling between the horizontal (or vertical) polarization ports of the unit pairs. The maximum mutual coupling occurs between vertical polarization ports of unit $\mathrm{B} 2$ and $\mathrm{C} 1$, and is less than $-35 \mathrm{~dB}$ over the operating bandwidth, which is less than the mutual coupling between units in the same sub-array and Class-A mutual coupling analyzed in Section III. This is because the adjacent sub-arrays are not in the same plane.

\section{CONCLUSION}

In this paper, a compact dual-polarized antenna with four radiating square patches has been designed with low mutual coupling. The mutual coupling between different ports were analyzed including ports exciting different polarization modes in the same antenna unit, and ports in different antenna units in the same and different sub-arrays. An array configuration containing 18 sub-arrays was constructed for practical considerations. With the closely placed adjacent patches and compact array configuration, the proposed Massive MIMO array only occupied a volume of about $8 \lambda \times 8 \lambda \times 3 \lambda$ and offered a maximum mutual coupling lower than $-35 \mathrm{~dB}$ between any two ports in the array. The proposed array could be a strong contender for the small cell base station deployment in the urban area with high-density buildings for $5 \mathrm{G}$ wireless systems.

\section{REFERENCES}

[1] G. J. Foschini, "Layered space-time architecture for wireless communication in a fading environment when using multi-element antennas," Bell Labs Tech. J., vol. 1, no. 2, pp. 41-59, 1996.
[2] Y. Gao, X. Chen, Z. Ying, and C. Parini, "Design and performance investigation of a dual-element pifa array at $2.5 \mathrm{ghz}$ for mimo terminal," IEEE Trans. Antennas Propag., vol. 55, no. 12, pp. 3433-3441, Dec. 2007.

[3] M. A. Jensen and B. K. Lau, "Uncoupled matching for active and passive impedances of coupled arrays in mimo systems," IEEE Trans. Antennas Propag., vol. 58, no. 10, pp. 3336-3343, Oct. 2010.

[4] H. Li, B. K. Lau, Z. Ying, and S. He, "Decoupling of multiple antennas in terminals with chassis excitation using polarization diversity, angle diversity and current control," IEEE Trans. Antennas Propag., vol. 60, no. 12, pp. 5947-5957, Dec. 2012.

[5] F. Rusek, D. Persson, B. K. Lau, E. G. Larsson, T. L. Marzetta, O. Edfors, and F. Tufvesson, "Scaling up mimo: Opportunities and challenges with very large arrays," IEEE Signal Process. Mag., vol. 30, no. 1, pp. 40-60, Jan. 2013.

[6] E. Larsson, O. Edfors, F. Tufvesson, and T. Marzetta, "Massive mimo for next generation wireless systems," IEEE Commun. Mag., vol. 52, no. 2, pp. 186-195, Feb. 2014.

[7] X. Pang, W. Hong, T. Yang, and L. Li, "Design and implementation of an active multibeam antenna system with $64 \mathrm{rf}$ channels and 256 antenna elements for massive mimo application in $5 \mathrm{~g}$ wireless communications," China Commun., vol. 11, no. 11, pp. 16-23, Nov. 2014.

[8] Y. Zeng, R. Zhang, and Z. N. Chen, "Electromagnetic lens-focusing antenna enabled massive mimo: Performance improvement and cost reduction," IEEE J. Sel. Areas Commun., vol. 32, no. 6, pp. 1194-1206, Jun. 2014.

[9] I. Tzanidis, B. L. Ng, and et. al, "Patch antenna array configuration for application in fd-mimo systems," in Proc. IEEE Antennas Propag. Soc. Int. Symp., Jul. 2013, pp. 2241-2242.

[10] O. P. Falade, M. U. Rehman, Y. Gao, X. Chen, and C. G. Parini, "Single feed stacked patch circular polarized antenna for triple band gps receivers," IEEE Trans. Antennas Propag., vol. 60, no. 10, pp. 44794484, Oct. 2012.

[11] S. Gao and A. Sambell, "Low-cost dual-polarized printed array with broad bandwidth," IEEE Trans. Antennas Propag., vol. 52, no. 12, pp. 3394-3397, Dec. 2004.

[12] W. Yun and Y. J. Yoon, "A wide-band aperture coupled microstrip array antenna using inverted feeding structures," IEEE Trans. Antennas Propag., vol. 53, no. 2, pp. 861-862, Feb. 2005.

[13] K. L. Wong and T. W. Chiou, "Finite ground plane effects on broadband dual polarized patch antenna properties," IEEE Trans. Antennas Propag., vol. 51, no. 4, pp. 903-904, Apr. 2003.

[14] H. Wong, K. L. Lau, and K. M. Luk, "Design of dual-polarized 1probe patch antenna arrays with high isolation," IEEE Trans. Antennas Propag., vol. 52, no. 1, pp. 45-52, Jan. 2004. 